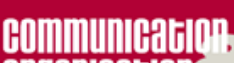
Oryanllatín.
Communication et organisation

Revue scientifique francophone en Communication organisationnelle

$52 \mid 2017$

Communauté, sociabilité et bien commun: approche internationale

\title{
La paix au pied du mur : quand Bogotá devient artiviste
}

The writing of peace is on the wall: when the city of Bogota becomes artivist

La paz como última opcion: cuando Bogotá se convierte en artivista

Quando a paz é a unica opção: quando Bogotá se torna artivista

\section{Oscar Motta Ramirez}

\section{OpenEdition}

1 Journals

\section{Édition électronique}

URL : https://journals.openedition.org/communicationorganisation/5688

DOI : 10.4000/communicationorganisation.5688

ISBN : 979-10-300-0257-7

ISSN : $1775-3546$

Éditeur

Presses universitaires de Bordeaux

Édition imprimée

Date de publication : 1 décembre 2017

Pagination : 99-112

ISBN : 979-10-300-0163-1

ISSN : $1168-5549$

\section{Référence électronique}

Oscar Motta Ramirez, «La paix au pied du mur : quand Bogotá devient artiviste », Communication et organisation [En ligne], 52 | 2017, mis en ligne le 01 décembre 2020, consulté le 02 janvier 2022. URL : http://journals.openedition.org/communicationorganisation/5688; DOI : https://doi.org/10.4000/ communicationorganisation.5688 


\title{
La paix au pied du mur : quand Bogotá devient artiviste
}

\author{
Oscar Motta Romirez ${ }^{1}$
}

\section{Introduction}

"La paix commence à être plus qu'un rêve, elle est plus proche que jamais » (Santos 2015) ${ }^{2}$. L'allocution vibrante du Président Santos, au soir de Noël 2015, ne laissait entrevoir un doute. Les négociations de paix menées de façon bilatérale depuis des années permettaient enfin d'envisager fermement un retour à la paix en Colombie après 52 années d'affrontements. Le gouvernement en place, la scène politique nationale, les FARC-EP, la communauté internationale, les leaders d'opinion, autant d'acteurs impliqués pour encourager un dialogue commun qui allait enfin aboutir durablement. Quelques mois plus $\operatorname{tard}^{3}$, à la surprise générale, les Colombiens ont pourtant rejeté par référendum l'accord signé entre le gouvernement et la guérilla, plongeant la Colombie dans l'incertitude absolue. Dans ce contexte controversé, des communautés de citoyens se sont spontanément constituées à travers tout le pays pour valoriser ce processus de paix et essayer de remobiliser la population par le biais de pratiques artistiques. Aussi, par une approche d'anthropologie socioculturelle, cet article s'appuie sur la présentation de deux études de cas comparatives, pour comprendre comment les facteurs culturels jouent dans les processus de transformation sociale. En effet, il s'agit ici d'analyser les procédés par lesquels les arts urbains ont repositionné de manière visible et active la population colombienne comme véritable coactrice du processus de paix. Pour mener notre analyse, nous nous sommes reposés sur des observations directes et indirectes de projets socioculturels

1 Oscar Motta est doctorant en Histoire de l'art auprès de l'université de Pau et des Pays de l'Adour. Rattaché au laboratoire ITEM (Identités, Territoires, Expressions, Mobilités, - EA 3002), sous la direction du Professeur Sabine Forero-Mendoza, il articule ses travaux de recherche autour de plusieurs axes de travail : les processus de création au travers des pratiques artistiques et culturelles, l'artivisme, la médiation culturelle, les territoires et liens sociaux ainsi que les nouveaux publics. Laboratoire ITEM, EA 3002, Université de Pau et des Pays de l'Adour ; oscarmotta@hotmail.com

2 Extrait en espagnol «La paz comienza a ser mucho más que un sueño y que está más próxima que nunca».

3 Référendum du 02 octobre 2016, appelant à se prononcer sur l'accord pour la fin du conflit et la construction d'une paix stable et durable entre le gouvernement et les Forces armées révolutionnaires de Colombie. 
qui se sont déroulés à Bogotá. Dans un premier temps, nous souhaitons mettre en exergue ces arts urbains qui se sont révélés être, dans un contexte de clivage politique et social fort en Colombie, un nouveau moyen d'expression pour ceux qui ne se sentaient pas entendus ou représentés dans cette victoire du non à l'accord de paix avec les FARC. Nous voulons ensuite analyser la manière dont la communauté, et tout particulièrement celle de Bogotá, s'est mobilisée et organisée pour proposer un réinvestissement créatif et esthétique de la contestation. Enfin nous souhaitons analyser comment les messages artivistes de la communauté sont devenus performatifs, créant de nouveaux espaces de socialisation et d'éducation, soutenus par les autorités publiques.

\section{Quand la communauté colombienne se saisit du processus de paix}

\section{Contexte général du conflit armé et du processus de paix}

La symbolique est forte. Le stylo utilisé pour signer le cessez-le-feu bilatéral et définitif a été fabriqué à partir d'une douille de balle. En ce jeudi 23 juin 2016, la guérilla des Fuerzas Armadas Revolucionarias de Colombia - Ejército del Pueblo $^{4}$ (FARC-EP) et le gouvernement colombien sont parvenus à sceller un accord de paix historique mettant fin à 52 années de guerre civile. Les quelques mots du Président Santos au moment de cette signature traduisent toute la tragédie de cet affrontement "Que cesse la nuit horrible de violence qui nous a couverts de son ombre durant plus d'un demi-siècle ! ${ }^{5}$. Enlèvements, prises d'otages, exécutions, attentats, mines antipersonnel : le bilan de ce conflit interne est dévastateur. En effet, 1073994 de morts, 167554 de disparus et 7243838 de déplacés sont aujourd'hui recensés ${ }^{6}$, plaçant ainsi la Colombie comme l'une des nations les plus touchées par ce fléau, juste derrière la Syrie 7 . Les raisons de ces violents affrontements demeurent complexes et ambiguës.

De multiples blocages sont imbriqués, trouvant entre autres leurs racines dans des enjeux politiques bipartistes, des négociations avortées, des logiques de vengeance féroces et des exclusions sociales au détriment des plus pauvres. Par ailleurs, il est important de souligner que pour tourner la page du conflit, d'âpres négociations ont été menées pour ratifier cet accord de paix et plusieurs points clés ${ }^{8}$ sont encore aujourd'hui discutés. L'un des aspects les

4 Traduit en français : «Les forces armées révolutionnaires de Colombie - Armée du peuple».

5 Extrait en espagnol: "¡Cesó la horrible noche de la violencia que nos ha cubierto con su sombra por más de medio siglo!".

6 Chiffres issus du Registre Unique des Victimes dédié au conflit armé colombien, URL : https://rni. unidadvictimas.gov.co/RUV.

7 Human Rights Watch, World Report 2014 Colombia, URL : https://www.hrw.org/world-report/2014/ country-chapters/colombia, Extrait en anglais : "More than 5 million Colombians have been internally displaced, and upward of 150,000 continue to flee their homes each year, generating the world's second largest population of internally displaced persons (IDPS)».

8 Texte du processus de paix (Acuerdo Final para la Terminación del Conflicto y la Construcción de una Paz Estable y Duradera), 24 novembre 2011, URL : http://www.altocomisionadoparalapaz.gov.co/procesos-y- 
plus sensibles concerne la volonté d'une réforme agraire en profondeur qui reconnaîtrait les droits des paysans, une meilleure accessibilité et répartition des terres et l'installation de services basiques en zones de conflit. L'ouverture $\mathrm{du}$ système politique colombien actuel est également remise directement en cause pour envisager une participation politique des FARC au débat démocratique avec l'attribution systématique de sièges au Congrès. Le processus de paix questionne également des stratégies de substitution des cultures illicites, afin de lutter contre le narcotrafic avec la coopération des FARC. Un autre point litigieux porte le désarmement des guérilleros et sur la responsabilité des crimes commis qui pourraient être minimisés par des amnisties, des peines alternatives ou des travaux au service de communautés sous certaines conditions.

\section{Détracteurs et frondeurs qui opacifient le processus de paix}

Ces éléments de contextualisation sont nécessaires pour comprendre les véritables impacts de l'accord de paix avec les FARC-EP sur le quotidien d'une majorité de Colombiens. En effet, même si les combats se sont éloignés depuis de nombreuses années des centres urbains, tels Bogotá, Medellin, Cali ou encore Barranquilla, de nombreuses régions du pays, beaucoup plus rurales, vont être directement concernées par les dispositions prises. Le cessez-le-feu négocié par le gouvernement colombien est globalement salué par la population. Cependant plusieurs voix se sont élevées dans la sphère publique pour se plaindre du manque de transparence sur les différents points de négociation du processus de paix, ce qui cristallise de nombreuses crispations dans le pays. Par exemple les médias, dont le discours le plus hostile est porté par la chaîne $\mathrm{RCN}$, s'appuient sur une approche testimoniale pour souligner l'animosité de la population qui conteste la réhabilitation des anciens guérilleros désireux de revenir à la vie civile dans leurs quartiers alors qu'ils y ont commis atrocités et crimes (RCN 2016). Les politiques, notamment au travers de chef du parti d'opposition et ex-président Álvaro Uribe, ne cessent de critiquer l'accord qu'ils jugent beaucoup trop édulcoré et dans lequel les guérilleros encourront des conditions judiciaires favorables, des aides financières de l'État alors que les victimes ne bénéficieraient que d'indemnisations inconsistantes (Uribe 2016). Plusieurs scientifiques ont également alerté sur les antagonismes soulevés par ces négociations et les réticences qu'elles génèrent. Jorge Restrepo, professeur d'économie de l'université Pontificia Universidad Javeriana, a clairement expliqué que le crime organisé a beaucoup à perdre si le processus de paix est ratifié. En effet, " la Colombie dispose d'un appareil militaire puissant qui consacre une grande part de son activité à la lutte contre les Farc. Si cette menace disparaît, le crime organisé deviendra le nouvel objectif des forces 
armées»(Restrero 2016). La philosophe Diana Uribe Forero a également démontré que ce processus de paix suscite de nombreuses incompréhensions dans la mesure où une certaine partie de l'opinion publique croit à tort que les négociations ne sont plus ouvertes et les décisions d'ores et déjà actées (Uribe Forero 2016).

\section{Les arts au-delà des mots}

Alors que dans ce contexte précis le pouvoir du langage se confronte à de nombreuses limites de compréhension et que les mots divisent plus la population qu'ils ne la fédèrent, la parole n'arrive plus à faire sens. Cette propagande d'hostilité a finalement conduit les Colombiens à refuser de peu le referendum ${ }^{9}$ sur l'accord avec la guérilla des FARC. Dans ce contexte d'incertitude absolue, des communautés citoyennes se sont pourtant spontanément constituées afin de renouveler les formes d'intervention en faveur du processus de paix qui venait d'être rejeté. Des anonymes, des cercles d'amis, des voisins, des quartiers entiers se sont mobilisés au travers de tout le pays pour offrir une nouvelle visibilité à la contestation. Cependant, celleci prit une forme beaucoup plus esthétique qu'elle ne l'était jusqu'à présent, s'appuyant sur des pratiques artistiques pour mobiliser différemment le grand public. Cette approche nous apparaît particulièrement intéressante à analyser. En effet, de nombreux auteurs scientifiques ont souligné la dimension dialogique des arts qui arrivent au travers des émotions qu'ils suscitent à abolir les frontières (Rizzi 2014, Arleo, Delalande 2011) et rassembler malgré les différences. D'autres complètent ce postulat et mettent en exergue le rôle majeur que les arts et la culture jouent dans les processus de transformation sociale.

L'expression artistique permet aux individus de parler d'eux-mêmes, de formuler des idées et des sentiments souvent difficiles à exprimer, et cela grâce aux codes et aux outils spécifiques proposés par la pratique artistique. La culture peut donc être un levier efficace pour la reconstruction d'identités individuelles et collectives, pour la restauration du lien social. (Auclair 2006)

Bousculantlescadresétablis,desinitiativeslocales,souventgéographiquement circonscrites, ont donc commencé à émerger afin de redonner la parole aux citoyens qui ne se sentaient pas entendus ou représentés. Toutes animées par l'envie de se réapproprier le débat public, elles visent à faire entendre la voix de chaque individu et à repositionner de manière visible et active la population comme véritable co-actrice de ce processus de paix.

9 Référendum du 02 octobre 2016, appelant à se prononcer sur l'accord pour la fin du conflit et la construction d'une paix stable et durable entre le gouvernement et les Forces armées révolutionnaires de Colombie. 


\title{
La recherche du commun par le biais du street art
}

\author{
Le street art à Bogotá: de l'idée politique à la légifération
}

D'étroites relations artistiques lient la France et la Colombie depuis plusieurs années. De nombreux auteurs ont souligné l'influence de la scène parisienne (Bascones, Quesada, Lorente 2009) sur le pays du réalisme magique de Gabriel García Márquez notamment au travers du mouvement de contestation étudiant qui a agité mai 68 (Tibble 2014). Les arts urbains ont trouvé à cette époque un contexte propice pour donner lieu à un nouveau mode d'expression transgressif et provocateur. La rue est alors devenue un lieu de débat public où les idées politiques pouvaient s'afficher et se manifester ( Les murs ont la parole », "L'imagination prend le pouvoir ! », « ORTF: La police vous parle tous les soirs à 20 heures ", etc.). Federico Calo a mis en exergue dans ses travaux la connexité qui lie naturellement le street art aux contextes de crise économique et sociale, montrant qu'il est l'expression de ces « crises urbaines » (Calo 2003). Les différences de classes sociales toujours plus marquées ont exacerbé les sentiments d'exclusion et de frustration des invisibles, qui ont choisi de s'exprimer dans la rue pour interpeller les institutions sur leur sort et bousculer les cadres établis. Alain Vulbeau (Vulbeau 1992) y décèle d'ailleurs une opportunité pour les jeunes en situation de précarité et de désocialisation de se valoriser. Aussi en 2011, quand le jeune graphiste Diego Felipe Becerra, tout juste âgé de 17 ans, fut iniquement abattu par la police nationale, alors qu'il était en train de peindre son personnage Félix le chat au bas d'un pont, il n'en fallut pas plus pour susciter l'indignation générale et transformer la contestation sociale grandissante en une véritable crise politique. Gustavo Petro, alors maire de Bogotá, fut obligé pour modérer les condamnations de l'ONU et apaiser les marches de protestation à répétition, de promulguer un décret (accord 482 de 2011 conduisant au décret 75 de 2013) décriminalisant l'art urbain et promouvant des pratiques responsables dans certains lieux spécifiques de la capitale. Dès lors, le tagging, les graffitis, ou encore les murales n'eurent de cesse de valoriser le dynamisme de la scène locale et de transformer la ville en un véritable terrain d'expérimentation sociale : "Le graffiti est un moyen de récupérer la ville, Bogotá c'est plus que des panneaux, des sans-abri et des voleurs... Le graffiti est un moyen non censuré qui peut permettre aux gens de récupérer du pouvoir » (Stinkfish 2015).

\section{La participation des habitants, un nouvel enjeu démocratique}

Si les crews ${ }^{10}$ sont les habituels acteurs d'une dynamique organisante structurée autour de codes, de rites et de règles précises, le processus de paix en Colombie fut le déclencheur d'une nouvelle relation co-construite par l'art urbain et la préoccupation sociétale. En effet, la participation citoyenne à la vie

10 Communautés de graffeurs qui se réunissent pour peindre ensemble. 
culturelle n'est pas un phénomène récent, mais elle retrouve dans ce contexte précis un nouvel enjeu démocratique. Comme l'indique Chloé Langeard, «les publics ne sont plus appréhendés comme de simples destinataires mais comme des acteurs à part entière du processus : il s'agit de "faire avec les habitants" et non plus simplement "pour les publics"» (Langeard 2015). Le projet "Share the word " s'est inscrit dans cette droite lignée et a trouvé un écho retentissant à Bogotá. La démarche initiale visait à peindre sur les murs des maisons des mots qui sont proposés par les habitants qui y résident. Ainsi à l'été 2016, l'Outsiders Krew a choisi Mariscal Sucre, quartier névralgique qui relie le cœur de la ville aux zones huppées du nord, pour réaliser une fresque colossale couvrant un ensemble de seize maisons. Le mot choisi par la communauté fut celui de «Paz» (paix) (Fig. 1). Aidés des habitants mais également d'étudiants appartenant à un programme de bénévolat socio-éducatif de l'université avoisinante Javeriana, plus d'une vingtaine de personnes ont contribué durant 40 jours à relier les aplats de couleurs aux immenses lettres blanches. Cette anamorphose est devenue le résultat d'une interaction entre les participants $\mathrm{du}$ projet, les artistes et l'environnement, faisant de l'art urbain un pont entre le point de vue de cette communauté et le monde extérieur. "C'est un mot qui a une consonance particulière pour des citoyens comme nous " (Share The Word Project 2016). «Plus que juste trois lettres, ça représente ce qu'on veut aujourd'hui en Colombie ${ }^{11}$. "La paix c'est plus que de simplement déposer des armes, c'est un changement de mentalité pour tout le monde $»^{12}$. Dans la mise en place de ce projet commun, l'habitant n'est plus considéré comme un simple usager, ni même un public potentiel. Une nouvelle identité ancrée au cœur du territoire lui est restituée, celle d'un citoyen à part entière qui se réapproprie son environnement, qui ré-ouvre le débat public et en offre un témoignage au monde extérieur.

\section{La participation des habitants, un travail de mémoire}

Si la participation des habitants permet au citoyen colombien de se réapproprier une légitimité collective dans le débat public, elle soulève également un autre enjeu. En effet, la recherche du commun peut enclencher un autre processus de transformation sociale et articuler l'intérêt général à celui des expressions individuelles. Au lendemain du rejet de l'accord de paix signé entre le gouvernement et les FARC, l'artiste plasticienne Doris Salcedo a appelé les habitants de Bogotá à la rejoindre autour d'un vaste projet de street installation. Afin de montrer la nécessité de mettre fin aux ravages causés par 52 années de guerre, elle a invité les habitants à coudre sur d'immenses draps blancs le nom des victimes du conflit, tel un linceul recouvrant symboliquement la Place Bolivar (Fig. 2). Sept kilomètres de tissu furent nécessaires 


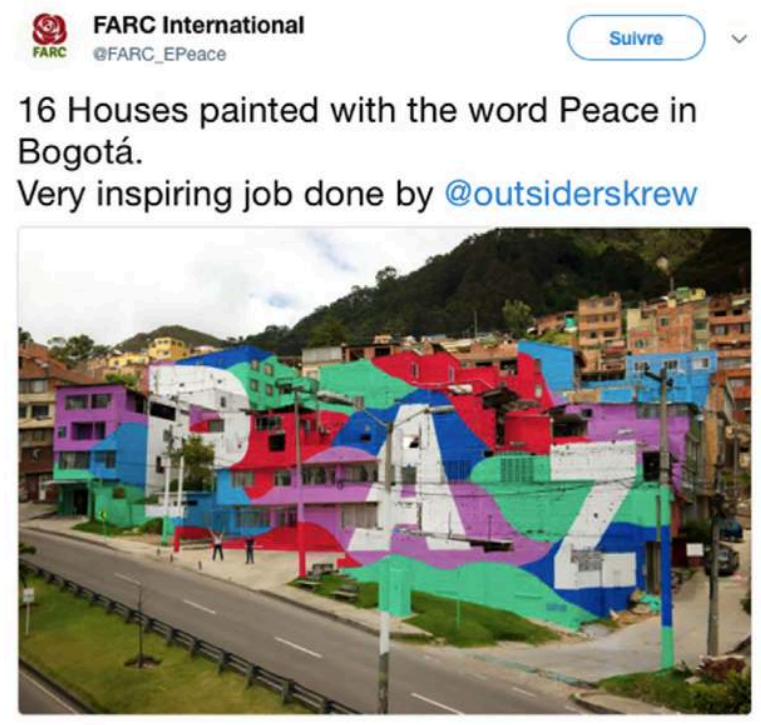

11:39 - 18 aoot 2016

Fig. 1 : Le projet « Share the word» identifié sur le compte twitter de FARC International, 18 août $2016^{13}$

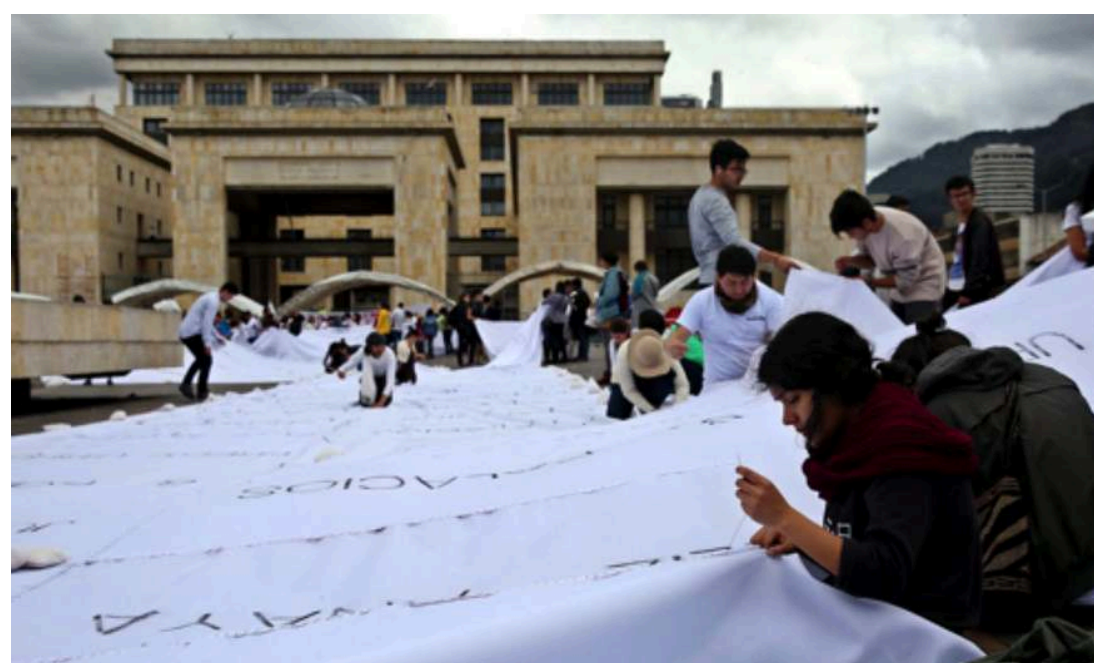

Fig. 2 : Le projet « Sumando ausencias », sur la place Bolivar, 12 octobre $2016^{14}$

13 Compte Twitter FARC International, 18 août 2016, URL : https://twitter.com/FARC_EPeace/ status/766343665258078208/photo/1?ref_src=twsrc\%5Etfw\&ref_url=https\%3A\%2F\%2Factu.fr\%2Fnorma ndie\%2Fcaen_14118\%2Fdeux-artistes-de-normandie-peignent-une-gigantesque-fresque-pour-la-paix-encolombie_762554.html

14 Nuevodiario.co, Sumando Ausencias, 12 octobre 2016, URL : http://www.nuevodiario.co/2016/10/12/ sumando-ausencias/ 
pour répondre à l'afflux de personnes. Accompagné dans la démarche par l'institut "La Unidad para la Atención y Reparación Integral a las Victimas", le projet "Sumando ausencias " se veut avant tout comme une intervention citoyenne dans laquelle la communauté propose un travail de mémoire où les victimes, leurs proches, et ceux qui s'identifient à leur cause, se mobilisent dans un même refus de l'oubli. Il s'agit ici de mettre en lumière de manière frappante les conséquences que cette tragédie a générées afin de faire prendre conscience de l'impératif de s'engager dans le processus de paix pour endiguer le fléau national. "Je ne sais pas pour l'art, je voulais juste participer à un hommage à ceux qui sont comme moi, et je voulais le faire avec ce que je sais et ce que j'ai » (Valencia 2016). "Je pense que c'est quelque chose de très symbolique, c'est une façon de dire aux Colombiens : nous pouvons être des sujets de droit, participer à ce type de choses qui n'appartiennent qu'à nous. Nous entrons dans un processus de réconciliation»(Ortiz 2016). Dans un tel contexte, la street installation ne se résume plus à la restitution finale qu'elle donne à voir mais s'accomplit dans la rétrospection individuelle qu'elle génère et la pacification quelle encourage parmi les héritiers d'un passé douloureux.

\section{L'artivisme ou l'art de la communauté devenue militante}

\section{De l'artiste militant à l'artivisme communautaire}

Depuis les années 2000, les actions hybrides d'art et d'activisme trouvent une légitimation sous le terme d'artivisme. Il est généralement caractérisé comme le regroupement "d'artistes ou groupes d'artistes [qui] s'organisent pour revendiquer, critiquer, lutter pour des droits, faisant appel à des pratiques éloignées des formes traditionnelles d'action politique et de leurs représentations institutionnalisées» (Do Nascimento Gonçalves 2009). L'artivisme se veut un art engagé où l'action est politique et la critique sociale, ce que Catherine Gingras définit avant tout comme un " art citoyen » (Gingras 2014). Mais il comporte une double finalité car il se veut également engageant. En effet, il cherche à « mobiliser le spectateur, à le sortir de son inertie supposée, à lui faire prendre position » (Bautes 2010). La plupart des travaux scientifiques font état du rôle joué par l'artiste dans cette démarche de contestation et soulignent l'importance des démarches individuelles pour initier de telles performances politiques. La pratique du street art s'inscrit dans cette même perspective populaire et militante et retrouve ses fondements dans la contestation callejer $a^{15}$ qui émergea en Amérique du sud dès les années 80. Les quartiers (barrios) y étaient identifiés comme des espaces de révolte (Tarragoni 2016) et le territoire est alors devenu un enjeu de pouvoir qu'il était impératif de marquer esthétiquement. Les peintures murales se sont

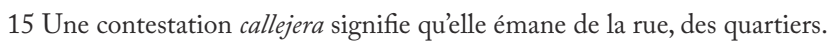


imposées comme l'expression de ces communautés constituées. De la même manière, les projets «Share the word» ou «Sumando ausencias » permettent aux habitants de recréer des espaces d'interpellation dans leur propre ville ou leur quartier, mais il est intéressant de noter que l'artivisme attaché au processus de paix en Colombie a fait évoluer une pratique individuelle classiquement observée chez l'artiste vers des dispositifs qui trouvent leurs forces dans le collectif. La communauté devient militante et ses messages socio-politiques prennent une forme performative. L'artiste ne fait que mettre à sa disposition des outils pour que son action interroge, détourne et interpelle. « L'artiste n'est plus seulement un créateur singulier d'œuvre d'art, il [devient] un créateur de lien social ».

\section{L'artivisme communautaire comme espace d'éducation}

Cet artivisme communautaire autour de demandes sociales civiques fortes et dans le même temps d'attentes personnelles singulières insuffle une dynamique particulière au sein de la collectivité nouvellement constituée. En effet, les projets cités sont la parfaite illustration de modes d'intervention profondément pédagogiques qui accompagnent les bénéficiaires (participants ou spectateurs) à acquérir de manière informelle de nouvelles valeurs, de nouvelles connaissances, de nouveaux comportements, de nouveaux espaces d'action, etc. La communauté devient en ce sens éducatrice et la ville se transforme en son cadre d'expérience. Le réinvestissement créatif des quartiers invite donc à " créer des savoirs ensemble » (Damon 1984). Ce travail artiviste fondamentalement collectif à Bogotá, enseigne à regarder différemment les problématiques sociétales rencontrées par les Colombiens. Plusieurs étapes participent de cet apprentissage et contribuent à cet enrichissement commun : la participation des habitants, la recherche d'une mixité des publics, l'élaboration de partenariats locaux, la notion de groupe de travail, le séquençage des ateliers, l'échange d'informations, la confrontation des idées, etc. La rue se donne à voir comme un espace de socialisation où la culture urbaine est créatrice de nouveaux savoirs citoyens. Pour Panitz, ces savoirs partagés trouvent leur fondement dans l'apprentissage collaboratif et constituent l'essence de l'esprit communautaire, à savoir « respecter et mettre en valeur les habiletés et contributions des membres du groupe " (Panitz 1999). Grâce à ces approches pédagogiques, que Baudrit décrit comme des " coordinations sociales de type partage » (Baudrit 2007), ce ne sont plus les individus qui font la démarche d'aller à l'art, mais bien la communauté mobilisée autour d'une question sociétale qui créé des situations, des contextes et des rencontres pour que l'art sensibilise les citoyens dans l'espace public. 


\section{De l'artivisme communautaire à l'institutionnalisation sociale}

Cet artivisme communautaire a su remplacer les débats politiques inopérants qui ne trouvaient plus écho dans la sphère publique auprès de la population colombienne. C'est par le partage de signes forts que les images ont renvoyé vers une mémoire collective et su actionner les leviers pertinents pour renouveler différemment la mobilisation autour du processus de paix. Leffervescence de cet art urbain (graffitis, installations, peintures murales, etc.) a reconnecté la rue et ses habitants, là où les pouvoirs publics n'arrivaient plus à être moteurs. De plus en plus plébiscitée et respectée par les communautés, cette « insurrection par les signes » (Baudrillard 1976) a interpellé les institutions en place dans la mesure où l'action modeste confinée à un niveau plutôt local, cet « éloge du petit » (Lindgaard 2005), trouvait pourtant un engouement au national voire bénéficiait d'une véritable médiatisation. Devant la spontanéité de ces nouvelles formes d'organisation et d'action critique, les autorités se sont interrogées sur la force de leur impact et la manière dont l'art urbain se révélait être un véritable instrument de conscientisation. Sans tomber dans les travers d'une récupération institutionnelle (Riffaud, Recours 2016) de cet artivisme qui aseptiserait le caractère contestataire des communautés, ni même une manipulation de la pratique artistique assujettie à une domination symbolique (Gibout, Leziard Yvon, Leziard Luc, 2006) du pouvoir en place, les autorités publiques colombiennes ont souhaité encourager ce mouvement citoyen et lui offrir une plus grande visibilité en officialisant dans un premier temps le "Bogotá Ciudad Memoria », un outil participatif cartographique qui permet à chacun d'identifier dans toute la ville des points de mémoire (71 actuellement), où l'art peut permettre de se souvenir des victimes et participer de manière active à la construction de la paix. Une artère principale au cœur du centre-ville a été rebaptisée "la plaza de los Murales de Memoria ", consacrée nationalement par la date du 9 avril et permet à chaque projet de street art porté par la communauté de s'exprimer à ciel ouvert.

\section{Conclusion}

Les formes d'intervention en faveur du processus de paix ont su se renouveler au travers des arts urbains en Colombie. Des communautés de citoyens se sont spontanément constituées pour essayer de remobiliser la population au lendemain du refus du referendum sur l'accord avec la guérilla des FARC. Le street art s'est imposé comme un art majeur dans cette contestation politique, notamment à Bogotá, créant des symboles communs qui favorisent la cohésion et le lien social. Ainsi la coopération autour de plusieurs projets artivistes s'est vue renforcée par l'expérience et le talent de chacun de ses participants. Là où de nombreux dispositifs gouvernementaux avaient échoué, ces modes d'intervention profondément pédagogiques ont ouvert de nouveaux espaces 
d'action qui pèsent aujourd'hui dans le débat public en Colombie. « Les rues sont le vrai champ de bataille de la démocratie [...] Je vois le street art comme un média alternatif. L'espace public est une arène intensément politique » (Pujas 2015 : 48). Les citoyens colombiens semblent avoir réussi à trouver un moyen de devenir acteurs et de jouer un rôle majeur dans leur processus de paix.

\section{BIBLIOGRAPHIE}

ARLEO Andy, DELALANDE Julie, Cultures enfantines, Presses universitaires de Rennes, 2011, 468 pages.

AUCLAIR Elizabeth, Offres et demandes culturelles, ou la spécificité du développement culturel en banlieue, in BERTUCCI Marie-Madeleine, HOUDARTMÉROT Violaine, Situations de banlieues, INRP, 2005, 290 pages.

AUCLAIR Elizabeth, Comment les arts et la culture peuvent-ils participer à la lutte contre les phénomènes de ségrégation dans les quartiers en crise ? Hérodote, vol. $3, \mathrm{n}^{\circ} 122$, 2006, p. 212-220.

BASCONES Pedro, El arte público como agente de revitalización urbana mediante la participación cuidadana, in QUESADA Blanca Fernández, LORENTE Jesús Pedro, Arte en el espacio públicobarrios artísticos y revitalización urbana, Prensas Universitarias de Zaragoza, 2009, p. 145-162.

BAUDRILLARD Jean, Kool killer: Les graffitis de New York ou l'insurrection par les signes, Gallimard, 1976, p. 118-128.

BAUTES Nicolas, L'expérience « artiviste » dans une favela de Rio de Janeiro, Cahiers de géographie du Québec, volume 54, numéro 153, 2010, p. 471-498.

CALO Federico, Le monde du graff, L'Harmattan, 2003, 146 pages.

DAMON William, Peer education: The untapped potential, Journal of Applied Developmental Psychology, 1984, p. 331-343.

GIBOUT Christophe, Techniques libératrices et techniques contraignantes : derrière le miroir des apparences..., in Yvon LEZIARD, Luc ROBENE, L'homme en mouvement, Chiron, 2006, p. 349-371.

PUJAS Sophie, Street art : poésie urbaine, Edi8, 2015, 192 pages.

RIZZI Alice Titia, Entre ici et là-bas, je vous dessine mon chez moi. Exploration qualitative des productions des enfants en psychothérapie transculturelle, Thèse de doctorat en Psychologie clinique, Université Paris Descartes, 2014, 389 pages.

VULBEAU Alain, Du tag au tag, Malakoff, 1992, 313 pages.

\section{Sitographie}

BAUDRIT Alain, Apprentissage coopératif/Apprentissage collaboratif: d'un comparatisme conventionnel à un comparatisme critique, Les Sciences de l'éducation Pour l'Ère nouvelle, 2007, Vol. 40, URL: https://www.cairn.info/revue-les-sciences-de-1education-pour-1-ere-nouvelle-2007-1-page-115.htm 
DO NASCIMENTO GONÇALVES Fernando, Art, activisme et le transpolitique : étude sur des utilisations artistiques des technologies de la communication en France et au Brésil, Sociétés, 2009, n 104, URL: https://www.cairn.info/revue-societes-2009-2page-115.htm

GINGRAS Catherine, Les arts : révéler, critiquer et transformer les rapports entre individus, environnement et ville, Environnement Urbain, 2014, URL : http://eue.revues. org/319

GRAVEREAU Sophie, Art et activisme dans le quartier parisien de Belleville, L'Information géographique, 2012, https://www.cairn.info/revue-1-informationgeographique-2012-3-p-52.htm

HUMAN RIGHT WATCH, World Report 2014 Colombia, URL : https://www.hrw. org/world-report/2014/country-chapters/colombia

LANGEARD Chloé, Les projets artistiques et culturels de territoire. Sens et enjeux d'un nouvel instrument d'action publique, Informations sociales, 2015, $\mathrm{n}^{\circ}$ 190, URL : https://www.cairn.info/revue-informations-sociales-2015-4-page-64.htm

LINDGAARD Jade, Artivisme, Vacarme, 2005, n 31, URL : https:/www.cairn.info/ revue-vacarme-2005-2-page-30.htm

ORTIZ Christian, Colombie: les noms de milliers de victimes du conflit brodés sur une toile blanche, Challenges, 12 octobre 2016, URL: https://www.challenges.fr/ monde/colombie-les-noms-de-milliers-de-victimes-du-conflit-brodes-sur-une-toileblanche_432484

PANITZ Theodore, Cooperative versus collaborative learning : A comparison of two concepts which will help us understand underlying nature of interactive learning, 1999, URL : https://files.eric.ed.gov/fulltext/ED448443.pdf

SANTOS Juan Manuel, Allocution du Président de la République de Colombie, 24 décembre 2015, URL : https://www.youtube.com/watch?v=15kW7rCOGRw

RCN, El acuerdo de paz está fundado sobre bases de impunidad, 25 août 2016, URL : http://www.rcnradio.com/nacional/acuerdo-paz-esta-fundado-bases-impunidad-josemiguel-vivanco/

RESTREPO Jorge, Jorge Restrepo dialoga sobre el acuerdo de paz en Colombia, $V O A$, URL : https://www.voanoticias.com/a/3480593.html, 25 août 2016.

RIFFAUD Thomas, RECOURS Robin, Le street art comme micro-politique de l'espace public : entre « artivisme » et coopératisme », Cabiers de Narratologie, numéro 30, 2016, URL : http://narratologie.revues.org/7484

SHARE THE WORD PROJECT, PAZ The story bebind the mural, 2016, URL : https://outsiderskrew.com/videos/

STINKFISH, Pochoirs et improvisation graphique dans les rues de Bogotà, Fatcap, 2015, URL : https://www.fatcap.org/article/810.html

TARRAGONI Federico, Le peuple est dans la rue. Politiques du street art dans les barrios vénézuéliens, Sociologie de l'Art, 2016, https://www.cairn.info/revue-sociologiede-1-art-2016-1-p-129.htm

TIBBLE Christopher, ¡A la calle!, Revue Arcadia, 2014, URL : http://www. revistaarcadia.com/impresa/arte/articulo/a-calle/39434 
URIBE Álvaro, Terrorismo triunfante ha logrado que le aprueben toda su agenda, PSCP TV, URL : https://www.pscp.tv/AlvaroUribeVel/1DXxyLReRIYJM 27 septembre 2016, Carthagène, Colombie.

URIBE FORERO Diana, Dejemos de matarnos : el espíritu del acuerdo de paz, Magic Markers, juin 2016, URL : https://www.youtube.com/watch?v=FojwIc-4_zU

VALENCIA Isaac, Las dudas que levantó la obra de Doris Salcedo en la Plaza de Bolívar, Vice, 12 octobre 2016, URL : https://www.vice.com/es_co/article/nnp87g/dorissalcedo-plaza-bolivar-victimas-sumando-ausencias

Résumé : 52 années de guerre civile ont ravagé la Colombie. Alors qu'un accord de paix venait d'être signé entre la guérilla des FARC et le gouvernement après plusieurs années de négociations, la population colombienne a pourtant rejeté par referendum cette ratification, plongeant le pays dans l'incertitude absolue. Seules 60000 voix ont fait l'écart. Tous les Colombiens ne se sont pas forcément sentis entendus ou représentés dans cette victoire du non. Aussi des communautés citoyennes (anonymes, cercles d'amis, voisins, quartiers entiers) se sont spontanément mobilisées afin de renouveler les formes d'intervention en faveur du processus de paix, par le biais de pratiques artistiques. Cet article vise dans un premier temps, à mettre en exergue les arts urbains qui se sont révélés être, dans ce contexte de clivage politique et social fort, un nouveau moyen d'expression. Il cherche également à analyser la manière dont la communauté, et tout particulièrement celle de Bogotá, s'est mobilisée et organisée pour proposer un réinvestissement créatif et esthétique de la contestation. Enfin il souhaite montrer comment les messages artivistes de la communauté sont devenus performatifs, créant de nouveaux espaces de socialisation et d'éducation.

Mots-clés : Colombie, Bogotá, FARC, guérilla, processus de paix, referendum, communautés, citoyens, contestation, arts urbains, street art, créativité, esthétique, artivisme, socialisation, éducation, institutionnalisation.

Abstract : 52 years of civil war ravaged Colombia. While a peace agreement had just been signed between the FARC guerrillas and the government after several years of negotiations, a substantial part of the Colombian population rejected this ratification by referendum, plunging the country into absolute uncertainty. Only 60,000 votes made the gap. All Colombians did not necessarily feel heard or represented in this result. Also citizen communities (anonymous, circles of friends, neighbors, whole districts) spontaneously mobilized to renew the forms of intervention for the peace process, through artistic practices. The aim of this article is first of all to highlight the urban arts which have proved to be, in this context of strong political and social divide, a new means of expression. It also seeks to analyze how the community, especially in Bogotá, mobilized and organized to propose a creative and aesthetic reinvestment of the protest. Finally, it wants to show how the artivist messages of the community became performative, creating new spaces of socialization and education. 
Keywords: Colombia, Bogotá, FARC, guerrilla, peace process, referendum, communities, citizens, protest, urban arts, street art, creativity, aesthetics, artivism, socialization, education, institutionalization.

Resumen: 52 años de guerra civil devastaron a Colombia. Mientras que un acuerdo de paz se acababa de firmar entre la guerrilla de las FARC y el gobierno después de varios años de negociaciones, la población colombiana rechazaba esta ratificación por referéndum, sumiendo al pais en una incertidumbre absoluta. Solo 60,000 votos hicieron la diferencia. Todos los colombianos no necesariamente se sintieron escuchados o representados en esta victoria del no. También las comunidades ciudadanas (anónimas, círculos de amigos, vecinos, barrios enteros) se movilizaron espontáneamente para renovar las formas de intervención para el proceso de paz, a través de prácticas artísticas. El objetivo de este artículo es, en primer lugar, resaltar las artes urbanas que han demostrado ser, en este contexto de fuerte división politica y social, un nuevo medio de expresión. También busca analizar cómo la comunidad, y especialmente la de Bogotá, se movilizó y organizó para proponer una reinversión creativa y estética de la protesta. Finalmente, quiere mostrar cómo los mensajes artivistas de la comunidad se han vuelto performativos, creando nuevos espacios de socialización y educación.

Palabras clave: Colombia, Bogotá, FARC, guerrilla, proceso de paz, referéndum, comunidades, ciudadanos, protesta, artes urbanas, arte callejero, creatividad, estética, artivismo, socialización, educación, institucionalización. 